\title{
Analysis of Recurrence Relations Generalized from the 4-Peg
} Tower of Hanoi*

\section{Akihiro MATSUURA ${ }^{\dagger a)}$, Member}

SUMMARY In this paper, we analyze recurrence relations generalized from the Tower of Hanoi problem of the form $T(n, \alpha, \beta)=\min _{1 \leq t \leq n}\{\alpha T(n-$ $t, \alpha, \beta)+\beta S(t, 3)\}$, where $S(t, 3)=2^{t}-1$ is the optimal total number of moves for the 3-peg Tower of Hanoi problem. It is shown that when $\alpha$ and $\beta$ are natural numbers, the sequence of differences of $T(n, \alpha, \beta)$ 's, i.e., $\{T(n, \alpha, \beta)-T(n-1, \alpha, \beta)\}$, consists of numbers of the form $\beta 2^{i} \alpha^{j}(i, j \geq 0)$ lined in the increasing order.

key words: Tower of Hanoi, 4-peg, recurrence relation, integer sequence

\section{Introduction}

The Tower of Hanoi puzzle with 3 pegs was invented by E. Lucas in 1883 [9]. He also presented 4-peg puzzle in 1889. In 1907, Dudeney reproduced the 4-peg puzzle as "The Reve's Puzzle" [3]. These problems and their variants have been used as an introductory example of recursive algorithms and they have also been studied widely in computer science and discrete mathematics [2], [5], [7], [8], [11]-[14]. Stockmeyer's survey [13] lists more than 300 references, not included textbooks and articles in other research areas such as psychology. In the simplest case of using 3 pegs and $n$ disks, the algorithm of first moving the upper $n-1$ disks to the intermediate peg, moving the bottom disk to the peg of destination, and finally moving the remaining $n-1$ disks to the destination, is the best possible and the total number of moves is $2^{n}-1$. Somewhat surprisingly, the optimal solution for the general Tower of Hanoi problem using $k(\geq 4)$ pegs and $n$ disks is not known yet. The best upper bound is obtained by the algorithms by Frame [5] and Stewart [11]. Their algorithms are rediscovered many times ([12] lists them). Furthermore, Klavžar et al. showed that seven different approaches to the multi-peg Tower of Hanoi problem, which include the ones by Frame and Stewart, are all equivalent [8]. On the other hand, the subexponential lower bound was first proved by Szegedy [14] and it was improved by Chen et al.[2]. Since the upper bound is believed to be the optimal, it is called the "presumed optimal" solution.

The Stewart's recursive algorithm for the 4-peg Tower of Hanoi is written as follows. For $1 \leq t \leq n$, consider the procedures of first moving the upper $n-t$ disks to the

Manuscript received March 31, 2010.

Manuscript revised July 19, 2010.

${ }^{\dagger}$ The author is with School of Science and Engineering, Tokyo Denki University, Saitama-ken, 350-0394 Japan.

*A preliminary version of the paper was presented at 5th SIAM Workshop on Analytic Algorithmics and Combinatorics (ANALCO08).

a)E-mail: matsu@ rd.dendai.ac.jp

DOI: 10.1587/transinf.E94.D.220 intermediate peg using the 4 pegs, moving the remaining $t$ disks to the destination using the available 3 pegs, and then moving the $n-t$ disks to the destination with the 4 pegs. The algorithm chooses the minimum one among them. When the total number of moves is denoted by $S(n, 4)$, the recurrence relation is written as

$$
S(n, 4)=\min _{1 \leq t \leq n}\{2 S(n-t, 4)+S(t, 3)\} .
$$

This recurrence relation is solved with the difference

$$
S(n, 4)-S(n-1,4)=2^{i-1}
$$

for $t_{i-1}<n \leq t_{i}$, where $t_{i}$ is the triangular number, i.e., $t_{i}=i(i+1) / 2$.

To clarify the combinatorial structures latent in this type of recurrence relation and to cope with a wider range of problems such as the Tower of Hanoi variants on graphs later discussed in Sect. 5, we investigate the general recurrence relation of the form

$$
\begin{aligned}
& T(0, \alpha, \beta)=0, \\
& T(n, \alpha, \beta)=\min _{1 \leq t \leq n}\{\alpha T(n-t, \alpha, \beta)+\beta S(t, 3)\}(n \geq 1),
\end{aligned}
$$

where $\alpha$ and $\beta$ are arbitrary natural numbers. $S(n, 4)$ is then written as $S(n, 4)=T(n, 2,1)$.

The main contribution of this paper is to exactly solve this relation for all natural numbers $\alpha$ and $\beta$. Suppose that $\left\{a_{n}\right\}_{n \geq 1}$ is the integer sequence which consists of numbers of the form $2^{i} \alpha^{j}(i, j \geq 0)$ lined in the increasing order. Then for $\alpha \geq 2$, the difference of $T(n, \alpha, \beta)$ 's is written using this sequence as

$$
T(n, \alpha, \beta)-T(n-1, \alpha, \beta)=\beta a_{n} .
$$

$T(n, \alpha, \beta)$ is then computed by summing up the differences. We note that when $\alpha=3, a_{n}$ increases as follows.

$$
a_{n}=1,2,3,4,6,8,9,12,16,18, \cdots .
$$

These numbers are called " 3 -smooth numbers" and are explored extensively in number theory in relation to the distribution of prime numbers [6] and new number representations [1], [4], [10].

The remaining of the paper is organized as follows: In Sect. 2, we state the main results. In Sect. 3, some fundamental properties on the sequence $\left\{2^{i} \alpha^{j}\right\}$ are investigated. In Sect. 4, a proof of the main theorem is given. Tower of Hanoi variants on graphs are discussed in Sect. 5. Finally, concluding remarks are given in Sect. 6. 


\section{Main Results}

\subsection{Linearity of $T(n, \alpha, \beta)$ on $\beta$}

We first show that $T(n, \alpha, \beta)$ is linear on the parameter $\beta$.

Theorem 1: For any natural numbers $\alpha$ and $\beta, T(n, \alpha, \beta)$ is linear on $\beta$. Namely,

$$
T(n, \alpha, \beta)=\beta T(n, \alpha, 1)
$$

holds.

Proof. By induction on $n$. When $n=0, T(0, \alpha, \beta)=0=$ $\beta T(0, \alpha, 1)$. Therefore, the equality holds.

Next, suppose that for $n \leq k$, the equality holds. Then by this assumption,

$$
\begin{aligned}
& T(k+1, \alpha, \beta) \\
= & \min _{1 \leq t \leq k+1}\{\alpha T(k+1-t, \alpha, \beta)+\beta S(t, 3)\} \\
= & \min _{1 \leq t \leq k+1}\{\alpha \beta T(k+1-t, \alpha, 1)+\beta S(t, 3)\} \\
= & \beta \min _{1 \leq t \leq k+1}\{\alpha T(k+1-t, \alpha, 1)+S(t, 3)\} \\
= & \beta T(k+1, \alpha, 1) .
\end{aligned}
$$

Therefore, the linearity of $T(n, \alpha, \beta)$ also holds for $n=k+1$.

This completes the proof.

We note that the linearity of $T(n, \alpha, \beta)$ also holds for any real number $\beta$.

\subsection{Explicit Formulas for $T(n, \alpha, 1)$ and $\Delta T(n, \alpha, 1)$}

Owing to Theorem 1 , it is enough to compute $T(n, \alpha, 1)$ instead of $T(n, \alpha, \beta)$. We consider the following recurrence relation for $T(n, \alpha, 1)$.

$$
\begin{aligned}
& T(0, \alpha, 1)=0, \\
& T(n, \alpha, 1)=\min _{1 \leq t \leq n}\{\alpha T(n-t, \alpha, 1)+S(t, 3)\}(n \geq 1) .
\end{aligned}
$$

Tables 1 and 2 show values of $T(n, 3,1)$ and $T(n, 4,1)$ for $1 \leq n \leq 10$. In the tables, $t_{\min }$ is the value of the argument with which the right-hand side of the recurrence relation (1) takes the minimum, and $\Delta T(n, \alpha, 1)$ is the difference of $T(n, \alpha, 1)$ 's. When $\alpha=3$, we observe that all the numbers

Table 1 The values of $t_{\min }, T(n, 3,1)$, and $\Delta T(n, 3,1)$.

\begin{tabular}{|c|c|c|c|c|c|c|c|c|c|c|}
\hline$n$ & 1 & 2 & 3 & 4 & 5 & 6 & 7 & 8 & 9 & 10 \\
\hline$t_{\min }$ & 1 & 2 & 2 & 3 & 3 & 4 & 4 & 4 & 5 & 5 \\
\hline$T(n, 3,1)$ & 1 & 3 & 6 & 10 & 16 & 24 & 33 & 45 & 61 & 79 \\
\hline$\Delta T(n, 3,1)$ & 1 & 2 & 3 & 4 & 6 & 8 & 9 & 12 & 16 & 18 \\
\hline
\end{tabular}

Table 2 The values of $t_{\min }, T(n, 4,1)$, and $\Delta T(n, 4,1)$.

\begin{tabular}{|c|c|c|c|c|c|c|c|c|c|c|}
\hline$n$ & 1 & 2 & 3 & 4 & 5 & 6 & 7 & 8 & 9 & 10 \\
\hline$t_{\min }$ & 1 & 2 & 2,3 & 3 & 3,4 & 4 & 4,5 & 4,5 & 5 & 5,6 \\
\hline$T(n, 4,1)$ & 1 & 3 & 7 & 11 & 19 & 27 & 43 & 59 & 75 & 107 \\
\hline$\Delta T(n, 4,1)$ & 1 & 2 & 4 & 4 & 8 & 8 & 16 & 16 & 16 & 32 \\
\hline
\end{tabular}

of the sequence $\left\{2^{i} 3^{j}\right\}_{i, j \geq 0}$ appear in the increasing order as differences of $T(n, 3,1)$ 's. When $\alpha=4, T(n, 4,1)$ takes the minimum at two values of $t_{\min }$ for some $n$, which is different from the case of $\alpha=3$.

For understanding the characteristics of $\Delta T(n, \alpha, 1)$ 's in a unified manner, we consider the following set of sequences. Let $p$ and $q$ be natural numbers and let $\left\{a_{n}\right\}_{n \geq 1}$ be the sequence of numbers of the form $p^{i} q^{j}(i, j \geq 0)$ which are lined in the increasing order. Here, we note that when $q=p^{l}$ for some integer $l, p^{i} q^{j}$ s such that $p^{i} q^{j}=p^{i^{\prime}} q^{j^{\prime}}$ and $(i, j) \neq\left(i^{\prime}, j^{\prime}\right)$ appear successively. Then the sequence of differences $\{\Delta T(n, \alpha, 1)\}$ is exactly of this form $\left\{p^{i} q^{j}\right\}$ for $p=2$ and $q=\alpha$. Namely, we show the following theorem.

Theorem 2: Let $\alpha$ be a natural number and let $\left\{a_{n}\right\}_{n \geq 1}$ be the number sequence which consists of numbers of the form $2^{i} \alpha^{j}(i, j \geq 0)$ lined in the increasing order. Then the sequence of difference of $T(n, \alpha, 1)$ 's is equal to $\left\{a_{n}\right\}_{n \geq 1}$. Namely, for $n \geq 1$, the difference is written as

$$
T(n, \alpha, 1)-T(n-1, \alpha, 1)=a_{n} .
$$

Combining Theorems 1 and 2 leads to the following corollary.

Corollary 1: Using the same sequence $\left\{a_{n}\right\}_{n \geq 1}$, for any natural numbers $n, \alpha$, and $\beta, T(n, \alpha, \beta)$ is computed as

$$
T(n, \alpha, \beta)=\beta \sum_{i=1}^{n} a_{i}
$$

\section{Properties of Sequence $\left\{2^{i} \alpha^{j}\right\}$}

In this section, we show some lemmata on the properties of the sequence $\left\{a_{n}\right\}=\left\{2^{i} \alpha^{j}\right\}$.

Lemma 1: Let $\alpha$ be any natural number such that $\alpha \geq 2$ and let $\left\{a_{n}\right\}_{n \geq 1}$ be the sequence of numbers of the form $2^{i} \alpha^{j}$ $(i, j \geq 0)$ lined in the increasing order. Then the following statements hold.

(i) When $\alpha \neq 2^{l}$ for any integer $l$, for any integer $j \geq 0$,

$$
\left|\left\{a_{n} \mid \alpha^{j}<a_{n}<\alpha^{j+1}\right\}\right|=\max \left\{i \mid i \in \mathbf{N}, 2^{i}<\alpha^{j+1}\right\} .
$$

(ii) When $\alpha=2^{l}$ for some integer $l$, for any integers $j$ and $k$ such that $j \geq 0$ and $0 \leq k \leq l-1$,

$$
\left|\left\{a_{n} \mid a_{n}=2^{k} \alpha^{j}\left(=2^{j l+k}\right)\right\}\right|=j+1 .
$$

Proof. (i) By induction on $j$. For $j \geq 0$, we define

$$
I_{j}:=\left\{a_{n} \mid \alpha^{j}<a_{n}<\alpha^{j+1}\right\} \text { and } i_{j}:=\max \left\{i \mid i \in \mathbf{N}, 2^{i}<\alpha^{j+1}\right\} .
$$

When $j=0$,

$$
I_{0}=\left\{a_{n} \mid 1<a_{n}<\alpha\right\}=\left\{2^{i} \mid i \in \mathbf{N}, 2^{i}<\alpha\right\} .
$$

Therefore, $\left|I_{0}\right|=i_{0}$.

Next, assume that $\left|I_{j}\right|=i_{j}$. Since $I_{j+1}=\left\{a_{n} \mid \alpha^{j+1}<\right.$ $\left.a_{n}<\alpha^{j+2}\right\}$ is the disjoint union of two sets $\left\{\alpha a_{n} \mid a_{n} \in I_{j}\right\}$ 
and $\left\{2^{i} \mid i \in \mathbf{N}, \alpha^{j+1}<2^{i}<\alpha^{j+2}\right\}$,

$$
\begin{aligned}
\left|I_{j+1}\right|= & \left|\left\{\alpha a_{n} \mid a_{n} \in I_{j}\right\}\right|+\left|\left\{2^{i} \mid \alpha^{j+1}<a_{k}<\alpha^{j+2}\right\}\right| \\
= & \left|I_{j}\right|+\left|\left\{2^{i} \mid i \in \mathbf{N}, \alpha^{j+1}<2^{i}<\alpha^{j+2}\right\}\right| \\
= & \max \left\{i \mid i \in \mathbf{N}, 2^{i}<\alpha^{j+1}\right\} \\
& +\left|\left\{2^{i} \mid i \in \mathbf{N}, \alpha^{j+1}<2^{i}<\alpha^{j+2}\right\}\right| \\
= & \left|\left\{2^{i} \mid i \in \mathbf{N}, 2^{i}<\alpha^{j+2}\right\}\right| \\
= & i_{j+1} .
\end{aligned}
$$

Therefore, (i) is shown.

(ii) When $\alpha=2^{l}$ for some positive integer $l$, the numbers in the sequence $\left\{2^{i} \alpha^{j}\right\}_{i, j \geq 0}$ are written as

$$
\begin{aligned}
2^{i} \alpha^{j}= & 1,2,2^{2}, \cdots, 2^{l-1}, \\
& 2^{l}, 2^{l}, 2^{l+1}, 2^{l+1}, \cdots, 2^{2 l-1}, 2^{2 l-1}, \\
& \cdots \\
& 2^{j l}, \cdots, 2^{j l}, \cdots, 2^{j l+k}, \cdots, 2^{j l+k}, \cdots, 2^{(j+1) l-1}, \\
& \cdots .
\end{aligned}
$$

For any $k$ such that $0 \leq k \leq l-1$, there are $j+1$ ways to obtain $2^{j l+k}$ using 2 and $\alpha\left(=2^{l}\right)$. Therefore, for any $k, 2^{j l+k}$ appears exactly $j+1$ times. Namely, the following holds.

$$
\left|\left\{a_{n} \mid a_{n}=2^{k} \alpha^{j}=2^{j l+k}\right\}\right|=j+1 .
$$

This completes the proof of Lemma 1.

Using Lemma 1, we show the following lemma which plays an important role to prove the main theorem.

Lemma 2: Let $\alpha$ be any natural number such that $\alpha \geq 2$ and let $\left\{a_{n}\right\}_{n \geq 1}$ be the sequence of numbers of the form $2^{i} \alpha^{j}$ $(i, j \geq 0)$ lined in the increasing order. Then the following statements hold.

(i) When $\alpha \neq 2^{l}$ for any integer $l$, for any $n$ such that $2^{i}<$ $a_{n}<2^{i+1}, a_{n}=\alpha a_{n-(i+1)}$.

(ii) When $\alpha=2^{l}$ for some integer $l$, for any $i$ and $n$ such that $a_{n}=2^{i}, a_{n+1}=\alpha a_{n-i}$.

Proof. (i) By induction on $i$. Let $i_{0}$ be the integer such that $2^{i_{0}-1}<\alpha<2^{i_{0}}$. Then since $\alpha>2, \alpha$ is the only number in $\left\{a_{n}\right\}$ such that $2^{i_{0}-1}<a_{n}<2^{i_{0}}$. Furthermore, $a_{i_{0}+1}=\alpha$ holds.

The base case is when $i=i_{0}-1$. In this case,

$$
\alpha a_{\left(i_{0}+1\right)-i_{0}}=\alpha a_{1}=\alpha .
$$

Therefore, $a_{i_{0}+1}=\alpha a_{\left(i_{0}+1\right)-i_{0}}$ holds.

Next, assume that for all $n$ such that $2^{t-1}<a_{n}<2^{t}$ and $1 \leq t \leq i, a_{n}=\alpha a_{n-t}$ holds. We show that for any $N$ such that $2^{i}<a_{N}<2^{i+1}, a_{N}=\alpha a_{N-(i+1)}$ holds. We divide into the following two cases: When $a_{N}=\alpha^{j}$ for some integer $j$ (Case 1); and otherwise (Case 2).

Case 1. When $a_{N}=\alpha^{j}$, by Lemma 1(i), there exist $i a_{n}$ 's between $\alpha^{j-1}$ and $a_{N}=\alpha^{j}$. So, $a_{N-(i+1)}=\alpha^{j-1}$. Therefore, $a_{N}=\alpha a_{N-(i+1)}$ holds.

Case 2. When $a_{N} \neq \alpha^{j}$ for any integer $j \geq 0, a_{N}$ is divisible by 2 . So, there exists some $M$ such that $2^{i-1}<a_{M}<2^{i}$ and $a_{N}=2 a_{M}$. Then by assumption of induction, $a_{M}=\alpha a_{M-i}$. Therefore,

$$
a_{N}=2 a_{M}=2\left(\alpha a_{M-i}\right)=\alpha\left(2 a_{M-i}\right) .
$$

To prove $a_{N}=\alpha a_{N-(i+1)}$, it is enough to show that $2 a_{M-i}=$ $a_{N-(i+1)}$. By the definition of $\left\{a_{n}\right\}$ and since $a_{M}<2^{i}<a_{N}$,

$$
\begin{aligned}
& \left|\left\{a_{n} \mid a_{M} / \alpha<a_{n}<a_{N} / \alpha\right\}\right| \\
& =\left|\left\{a_{n} \mid a_{M}<a_{n}<a_{N}\right\}\right|-1 \\
& =(N-M-1)-1 \\
& =N-M-2 .
\end{aligned}
$$

We note that at the first equality, -1 comes from the deletion of $2^{i}$. Using this equality with $a_{M} / \alpha=a_{M-i}, a_{N} / \alpha$ is computed as

$$
a_{N} / \alpha=a_{(M-i)+(N-M-2)+1}=a_{N-(i+1)} .
$$

Therefore, $a_{N}=\alpha a_{N-(i+1)}$ holds.

This completes the proof for (i).

(ii) Suppose that $\alpha=2^{l}$ for some positive integer $l$. For $j \geq 0$ and $0 \leq k \leq l-1$, let $G_{j, k}$ be the subsequence of $\left\{a_{n}\right\}$ which consists of all of the identical $2^{j l+k}$ 's. Namely,

$$
G_{j, k}=\left\{2^{j l+k}, 2^{j l+k}, \cdots, 2^{j l+k}\right\} .
$$

By Lemma 1(ii), $\left|G_{j, k}\right|=j+1$ for any $j$ and $k$ such that $j \geq 0$ and $0 \leq k \leq l-1$. We further define the subsequence $G_{j}$ for $j \geq 0$ by the union of subsequence $G_{j, k}$ 's over $0 \leq k \leq l-1$. Namely,

$$
\begin{aligned}
G_{j} & =\bigcup_{k=0}^{l-1} G_{j, k} \\
& =\left\{2^{j l}, \cdots, 2^{j l}, \cdots, 2^{j l+k}, \cdots, 2^{j l+k}, \cdots, 2^{(j+1) l-1}\right\} .
\end{aligned}
$$

Now, it is enough to show that for any $a_{n}=2^{j l+k}$ in $G_{j}$, $a_{n+1}=\alpha a_{n-(j l+k)}$ holds.

Suppose that $a_{n}=2^{j l+k}$ is an arbitrary element in $G_{j}$. Then there is some $h, 1 \leq h \leq j+1$ such that $a_{n}=2^{j l+k}$ is the $h$ th element in the sequence $G_{j, k}$. (Recall that all the elements in $G_{j, k}$ are the same numbers.) In this case, $n$ and $n-(j l+k)$ are expressed as follows.

$$
\begin{aligned}
n & =\sum_{m=1}^{j}\left|G_{m}\right|+(j+1) k+h \\
& =\sum_{m=1}^{j} m l+(j+1) k+h \\
& =\frac{l j(j+1)}{2}+(j+1) k+h . \\
n-(j l+k) & =\frac{l j(j+1)}{2}+(j+1) k+h-(j l+k) \\
& =\frac{l(j-1) j}{2}+j k+h \\
& =\sum_{m=1}^{j-1}\left|G_{m}\right|+j k+h .
\end{aligned}
$$


Since the subsequence where $a_{n-(j l+k)}$ is located differs according to the value of $h$, we divide into the following two cases: When $1 \leq h \leq j$ (Case 1 ); and when $h=j+1$ (Case 2).

Case 1. When $1 \leq h \leq j, a_{n-(j l+k)}$ is the $h$ th element in $G_{j-1, k}$. So, $a_{n-(j l+k)}=2^{(j-1) l+k}$. Therefore,

$$
\alpha a_{n-(j l+k)}=2^{l} 2^{(j-1) l+k}=2^{j l+k}=a_{n+1} .
$$

Case 2. When $h=j+1, a_{n}$ is the last element in $G_{j, k}$. Then, $a_{n-(j l+k)}$ is the first element in $G_{j-1, k+1}$, i.e., $2^{(j-1) l+k+1}$, except for the case $a_{n}=2^{(j+1) l-1}$ is the last element in $G_{j}$. We consider this exceptional case later. Since $a_{n-(j l+k)}=$ $2^{(j-1) l+k+1}$,

$$
\alpha a_{n-(j l+k)}=2^{l} 2^{(j-1) l+k+1}=2^{j l+k+1}=a_{n+1} .
$$

The last equality holds because $a_{n}$ is the last element $\left(2^{j l+k}\right)$ in $G_{j, k}$.

We finally consider the exceptional case, that is, when $a_{n}=2^{(j+1) l-1}$ is the last element in $G_{j}$. In this case, $k=l-1$, so $a_{n-(j l+k)}=a_{n+1-(j+1) l}$. Since $\left|G_{j}\right|=(j+1) l$ and $a_{n+1}$ is the first element in $G_{j+1}, a_{n+1-(j+1) l}$ is the first element in $G_{j}$, i.e., $2^{j l}$. Therefore,

$$
\alpha a_{n-(j l+k)}=2^{l} 2^{j l}=2^{(j+1) l}=a_{n+1} .
$$

This completes the proof of Lemma 2.

\section{Proof of Theorem 2}

In this section, we prove Theorem 2 .

First, when $\alpha=1,\left\{a_{n}\right\}_{n \geq 1}=\left\{2^{i} 1^{j}\right\}_{i, j \geq 0}=\{1\}$. Namely, $a_{n}=1$ for all $n \geq 1$. On the other hand, $T(n, 1,1)=$ $\min _{1 \leq t \leq n}\{1 T(n-t, 1,1)+S(t, 3)\}$ takes the minimum at $t=1$ for all $n$ and it is computed as

$$
T(n, 1,1)=T(n-1,1,1)+S(1,3)=T(n-1,1,1)+1 .
$$

Therefore, $T(n, 1,1)-T(n-1,1,1)=a_{n}$ holds for all $n \geq 1$.

When $\alpha \geq 2$, the proof is divided into the following two cases: When $\alpha$ is not of the form $2^{l}$ for any positive integer $l$ (Case 1); and otherwise (Case 2).

Case 1. By induction on $n$.

When $n=1, a_{1}=2^{0} \alpha^{0}=1$. On the other hand, since $T(0, \alpha, 1)=0$ and $T(1, \alpha, 1)=\alpha T(0, \alpha, 1)+S(1,3)=$ $0+\left(2^{1}-1\right)=1, T(1, \alpha, 1)-T(0, \alpha, 1)=1$. Therefore, $T(1, \alpha, 1)-T(0, \alpha, 1)=a_{1}$ holds.

When $n \geq 2$, for $i \geq 0$, let $k_{i}$ be the integer such that $a_{k_{i}}=2^{i}$. We assume that the following equations hold for all $n$ such that $1 \leq n \leq k_{i}$.

$$
T(n, \alpha, 1)-T(n-1, \alpha, 1)=a_{n} .
$$

We show that the same equation holds for each of $n$ 's such that $k_{i}+1 \leq n \leq k_{i+1}$. For brevity, we define

$$
T_{n, t}:=\alpha T(n-t, \alpha, 1)+S(t, 3) .
$$

Then $T(n, \alpha, 1)=\min _{1 \leq t \leq n}\left\{T_{n, t}\right\}$.

We first clarify with which argument $T_{n, t}$ is minimized.
Lemma 3: Under the assumption of the induction, for all $n$ such that $k_{i} \leq n<k_{i+1}, T(n, \alpha, 1)=\min _{1 \leq t \leq n}\left\{T_{n, t}\right\}$ takes the minimum at $t=i+1$.

Proof. The difference $T_{n, t+1}-T_{n, t}$ is computed as follows.

$$
\begin{aligned}
& T_{n, t+1}-T_{n, t} \\
= & \{\alpha T(n-(t+1), \alpha, 1)+S(t+1,3)\} \\
& -\{\alpha T(n-t, \alpha, 1)+S(t, 3)\} \\
= & -\alpha\{T(n-t, \alpha, 1)-T(n-t-1, \alpha, 1)\} \\
& +\left(2^{t+1}-1\right)-\left(2^{t}-1\right) \\
= & -\alpha a_{n-t}+2^{t} \text { (by Assumption (2)). }
\end{aligned}
$$

(i) When $k_{i} \leq n<k_{i+1}-1$, we first show that for $t<i+1$, $T_{n, t}$ is monotonically decreasing. At Eq. (3), when $t<i+1$, both $-a_{n-t}$ and $2^{t}$ take the maximums at $t=i$. Therefore,

$$
\begin{aligned}
T_{n, t+1}-T_{n, t} & \leq-\alpha a_{n-i}+2^{i} \\
& <-\alpha a_{k_{i}-i}+2^{i} \quad\left(\text { since } k_{i} \leq n\right) \\
& =-a_{k_{i}+1}+a_{k_{i}} \quad(\text { by Lemma } 2(\mathrm{i})) \\
& <0 .
\end{aligned}
$$

Thus, $T_{n, t}$ is monotonically decreasing when $t<i+1$. We note that in showing this, Lemma 2 in the previous section is used.

When $t \geq i+1$, both $-a_{n-t}$ and $2^{t}$ take the minimums at $t=i+1$. Therefore,

$$
\begin{aligned}
T_{n, t+1}-T_{n, t} & \geq-\alpha a_{n-(i+1)}+2^{i+1} \\
& \geq-\alpha a_{k_{i+1}-1-(i+1)}+a_{k_{i+1}} \quad\left(\text { since } n<k_{i+1}\right) \\
& =-a_{k_{i+1}-1}+a_{k_{i+1}} \quad(\text { by Lemma } 2(\mathrm{i})) \\
& >0 .
\end{aligned}
$$

Thus, $T_{n, t}$ is monotonically increasing when $t \geq i+1$. Consequently, when $k_{i} \leq n<k_{i+1}, T_{n, t}$ takes the minimum at $t=i+1$.

This completes the proof of Lemma 3.

Now we are ready to prove Case 1 of Theorem 2 . It is further divided into two subcases: When $k_{i}+1 \leq n<k_{i+1}$ (Case 1-1); and when $n=k_{i+1}$ (Case 1-2).

Case 1-1. By Lemmata 2 and $3, T(n, \alpha, 1)-T(n-1, \alpha, 1)$ is computed for $k_{i}+1 \leq n<k_{i+1}$ as follows.

$$
\begin{aligned}
& T(n, \alpha, 1)-T(n-1, \alpha, 1) \\
= & T_{n, i+1}-T_{n-1, i+1} \\
= & \alpha\{T(n-(i+1), \alpha, 1)-T(n-1-(i+1), \alpha, 1)\} \\
& +S(i+1,3)-S(i+1,3) \\
= & \alpha a_{n-(i+1)} \quad(\text { by Assumption }(2)) \\
= & a_{n} \text { (by Lemma 2(i)). }
\end{aligned}
$$

Thus, Case 1-1 is shown.

Case 1-2. When $n=k_{i+1}$, we should prove $T\left(k_{i+1}, \alpha, 1\right)-$ $T\left(k_{i+1}-1, \alpha, 1\right)=a_{k_{i+1}}\left(=2^{i+1}\right)$. By Lemma 3, $T\left(k_{i+1}, \alpha, 1\right)$ and $T\left(k_{i+1}-1, \alpha, 1\right)$ take the minimums at $t=i+2$ and $t=i+1$, respectively. Therefore, 


$$
\begin{aligned}
& T\left(k_{i+1}, \alpha, 1\right)-T\left(k_{i+1}-1, \alpha, 1\right) \\
= & T_{k_{i+1}, i+2}-T_{k_{i+1}-1, i+1} \\
= & \alpha\left\{T\left(k_{i+1}-(i+2), \alpha, 1\right)-T\left(k_{i+1}-1-(i+1), \alpha, 1\right)\right\} \\
& +S(i+2,3)-S(i+1,3) \\
= & \left(2^{i+2}-1\right)-\left(2^{i+1}-1\right)=2^{i+1} .
\end{aligned}
$$

Thus, Case 1-2 is shown.

This completes the proof for Case 1.

Case 2. Now $\alpha=2^{l}$ for some integer $l \geq 1$. Similarly to Case 1 , we proceed by induction on $n$. For $i \geq 0$, let $k_{i}$ be the largest index $n$ such that $a_{n}=2^{i}$.

When $n=0$, the proof is exactly the same with Case 1 .

When $n \geq 1$, we assume that the following equation holds for all $n$ such that $1 \leq n \leq k_{i}$.

$$
T(n, \alpha, 1)-T(n-1, \alpha, 1)=a_{n} .
$$

We again extend these equations to $k_{i}+1 \leq n \leq k_{i+1}$; namely, for all $n$ such that $a_{n}=2^{i+1}$. Similarly to Lemma 3, we clarify with which argument $T_{n, t}$ is minimized by the following lemma.

Lemma 4: Under the assumption of induction, the following statements hold.

(i) When $n=k_{i}, T(n, \alpha, 1)=\min _{1 \leq t \leq n}\left\{T_{n, t}\right\}$ takes the minimum at $t=i+1$.

(ii) When $k_{i}+1 \leq n<k_{i+1}, T(n, \alpha, 1)=\min _{1 \leq t \leq n}\left\{T_{n, t}\right\}$ takes the minimum at $t=i+1$ and $t=i+2$.

Proof. Similarly to Lemma 3, we compute the difference $T_{n, t+1}-T_{n, t}=-\alpha a_{n-t}+2^{t}$.

(i) When $n=k_{i}$, we first show that when $t<i+1, T_{n, t}$ is monotonically decreasing. When $t<i+1$, both $-a_{n-t}$ and $2^{t}$ take the maximums at $t=i$. Therefore,

$$
\begin{aligned}
T_{k_{i}, t+1}-T_{k_{i}, t} & \leq-\alpha a_{k_{i}-i}+2^{i} \\
& =-a_{k_{i}+1}+a_{k_{i}} \text { (by Lemma 2(ii)) } \\
& <0 .
\end{aligned}
$$

Thus, $T_{k_{i}, t}$ is monotonically decreasing when $t<i+1$.

When $t \geq i+1$, both $-a_{n-t}$ and $2^{t}$ take the minimums at $t=i+1$. Therefore,

$$
\begin{aligned}
T_{k_{i}, t+1}-T_{k_{i}, t} & \geq-\alpha a_{k_{i}-(i+1)}+2^{i+1} \\
& >-\alpha a_{k_{i}-i}+2^{i+1} \\
& =-a_{k_{i}}+2^{i+1} \quad(\text { by Lemma 2(ii)) } \\
& =-2^{i}+2^{i+1} \\
& >0
\end{aligned}
$$

Thus, $T_{k_{i}, t}$ is monotonically increasing when $t \geq i+1$
when $n=k_{i}, T_{k_{i}, t}$ takes the minimum at $t=i+1$.

(ii) When $k_{i}+1 \leq n<k_{i+1}$, we note that $a_{n}=2^{i+1}$ constantly by definition of $k_{i}$. When $t<i+1$, both $-a_{n-t}$ and $2^{t}$ take the maximums at $t=i$. Therefore,

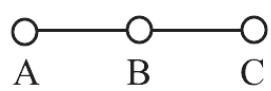

$$
\begin{aligned}
T_{n, t+1}-T_{n, t} & \leq-\alpha a_{n-i}+2^{i} \\
& =-a_{n+1}+2^{i} \quad(\text { by Lemma 2(ii)) } \\
& <-a_{k_{i}}+2^{i} \\
& =0 .
\end{aligned}
$$

Thus, $T_{n, t}$ is monotonically decreasing when $t<i+1$.

When $t=i+1, T_{n, t+1}-T_{n, t}$ is computed as

$$
\begin{aligned}
T_{n, i+2}-T_{n, i+1} & =-\alpha a_{n-(i+1)}+2^{i+1} \\
& =-\alpha a_{(n-1)-i}+2^{i+1} \\
& =-a_{n}+2^{i+1} \quad \text { by Lemma 2(ii)) } \\
& =0 .
\end{aligned}
$$

Therefore, $T_{n, i+2}=T_{n, i+1}$ holds.

When $t>i+1$, both $-a_{n-t}$ and $2^{t}$ take the minimums at $t=i+2$. Therefore,

$$
\begin{aligned}
T_{n, t+1}-T_{n, t} & \geq-\alpha a_{n-(i+2)}+2^{i+2} \\
& >-\alpha a_{n-i}+2^{i+2} \\
& =-a_{n+1}+2^{i+2} \text { (by Lemma 2(ii)) } \\
& \geq 0 .
\end{aligned}
$$

Thus, $T_{n, t}$ is monotonically increasing when $t>i+1$. In all, when $k_{i}+1 \leq n<k_{i+1}, T_{k_{i}, t}$ takes the minimum at $t=i+1$ and $t=i+2$.

This completes the proof of Lemma 4.

Now we are ready to prove Case 2 of Theorem 2, that is, $T(n, \alpha, 1)-T(n-1, \alpha, 1)=a_{n}$ for $k_{i}+1 \leq n \leq k_{i+1}$.

When $k_{i}+1 \leq n \leq k_{i+1}$, by Lemma $4, T_{n, t}$ takes the minimum at least at $t=i+2$ and $T_{n-1, t}$ takes the minimum at least at $t=i+1$. Therefore, for $k_{i}+1 \leq n \leq k_{i+1}$,

$$
\begin{aligned}
& T(n, \alpha, 1)-T(n-1, \alpha, 1) \\
= & T_{n, i+2}-T_{n-1, i+1} \\
= & \alpha\{T(n-(i+2), \alpha, 1)-T(n-1-(i+1), \alpha, 1)\} \\
& +\left(2^{i+2}-1\right)-\left(2^{i+1}-1\right) \\
= & 2^{i+1} .
\end{aligned}
$$

Thus, the proof for Case 2 is shown.

This completes the proof of Theorem 2 .

\section{Tower of Hanoi Variants on Graphs}

One of the motivation for considering the recurrence relation for $T(n, \alpha, \beta)$ is that they appear in the Tower of Hanoi variants on graphs. For example, let us consider the Tower of Hanoi problems on the graphs shown in Fig. 1. Now pegs

(b)

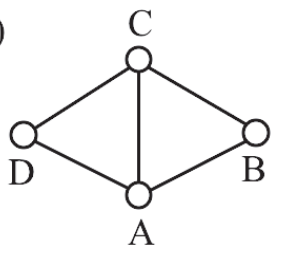

Fig. 1 Tower of Hanoi variants on graphs. 
are located on the vertices and a topmost disk of a peg is allowed to be moved to another peg only when there is an edge between the two pegs. The purpose of the problem for the graph in Fig. 1 (a) (Fig. 1 (b), resp.) is to move all the $n$ disks from A to $\mathrm{C}$ (A to B, resp.). Then these problems admit algorithms with the following recurrence relations, respectively.

$$
\begin{gathered}
\left\{\begin{array}{l}
T_{1}(0,3,2)=0 \\
T_{1}(n, 3,2)=3 T_{1}(n-1,3,2)+2(n \geq 1)
\end{array}\right. \\
\left\{\begin{array}{l}
T_{2}(0,3,1)=0 \\
T_{2}(n, 3,1)=\min _{1 \leq t \leq n}\left\{3 T_{2}(n-t, 3,1)+S(t, 3)\right\}(n \geq 1)
\end{array}\right.
\end{gathered}
$$

At the first example, smaller $n-1$ disks are moved by the procedure using $T_{1}(n-1,3,2)$ moves three times and the largest disk needs two more moves, so the recurrence relation for $T_{1}$ having $\alpha=3$ and $\beta=2$ holds in this case. At the second example, the procedure using $T_{2}(n-t, 3,1)$ moves are applied three times and the procedure using $S(t, 3)$ moves is applied once. Then the recurrence relation for $T_{2}$ having $\alpha=3$ and $\beta=1$ holds. Thus, the method and results for $T(n, \alpha, \beta)$ in the previous sections can be used for analyzing algorithms for the Tower of Hanoi variants on graphs.

\section{Concluding Remarks}

We made exact analysis of recurrence relations generalized from the 4-peg Tower of Hanoi problem. The differences of $T(n, \alpha, \beta)$ 's are shown to have unexpectedly simple form such as $\left\{2^{i} \alpha^{j}\right\}$. It has to be noted that the results of this paper are not the one to improve the bounds of the original multi-peg Tower of Hanoi problem. Rather, the contribution should lie on clarifying some combinatorial structures of recurrence relations generalized from the recursive algorithm for the Tower of Hanoi problem. Relationships of the results of this paper with number theory, especially on smooth numbers and on the sequences $\left\{p^{i} q^{j}\right\}$, should be of further interest.

\section{References}

[1] R. Blecksmith, M. McCallum, and J.L. Selfridge, "3-smooth representations of integers," Amer. Math. Monthly, vol.105, pp.529-543, June-July 1998.

[2] X. Chen and J. Shen, "On the Frame-Stewart conjecture about the Towers of Hanoi," SIAM J. Comput., vol.33, pp.584-589, 2004.

[3] H.E. Dudeney, The Reve's Puzzle, The Canterbury Puzzles (and Other Curious Problems), Thomas Nelson and Sons, London, 1907.

[4] P. Erdős, "Problem Q814," Math. Magazine, vol.67, pp.67, 74, 1994.

[5] J.S. Frame, "Solution to advanced problem 3918," Amer. Math. Monthly, vol.48, pp.216-217, 1941.

[6] G.H. Hardy and S. Ramanujan, "On the normal number of prime factors of a number $n$," Quart. J. Math. Oxford Ser., vol.48, pp.7692, 1917.

[7] S. Klavžar and U. Milutinović, "Simple explicit formula for the Frame-Stewart numbers," Ann. Combin., vol.6, pp.157-167, 2002.

[8] S. Klavžar, U. Milutinović, and C. Petr, "On the Frame-Stewart algorithm for the multi-peg Tower of Hanoi problem," Discr. Math., vol.120, pp.141-157, 2002.
[9] E. Lucas, Récréation Mathématiques, vol.III, Gauthier-Villars, Paris, 1893.

[10] N.J.A. Sloane, The On-Line Encyclopedia of Integer Sequences, http://www.research.att.com/njas/sequences, 1996-2005.

[11] B.M. Stewart, "Solution to advanced problem 3918," Amer. Math. Monthly, vol.48, pp.217-219, 1941.

[12] P.K. Stockmeyer, "Variations on the four-post Tower of Hanoi puzzle," Congr. Numer., vol.102, pp.3-12, 1994.

[13] P.K. Stockmeyer, "The Tower of Hanoi: A bibliography," manuscript, 1997-2005. Available at http://www.cs.wm.edu/ /pkstoc/h_papers.html

[14] M. Szegedy, "In how many steps the $k$ peg version of the Towers of Hanoi game can be solved?," Proc. STACS 99, LNCS 1563, pp.356$361,1999$.

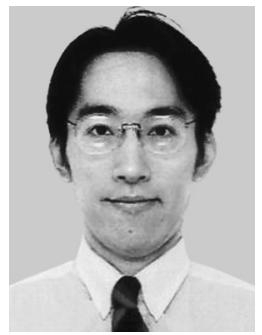

Akihiro Matsuura was born in 1968. He received his B.S. and M.S. degrees in mathematics from Kyoto University in 1992 and 1994, respectively and his $\mathrm{Ph} . \mathrm{D}$. in informatics from Kyoto University in 2002. From 1994 to 1999, he was with NTT Communication Science Laboratories. In 2003, he joined Tokyo Denki University where since 2008 he has been an associate professor. His research interests include combinatorics, algorithm theory, and game informatics. 